\title{
Common fixed point of four maps in S-metric spaces
}

\author{
S. Sedghi ${ }^{1} \cdot$ N. Shobkolaei ${ }^{2} \cdot$ M. Shahraki ${ }^{1} \cdot$ T. Došenovic $^{3}$
}

Received: 22 June 2017 / Accepted: 2 April 2018/Published online: 25 April 2018

(C) The Author(s) 2018

\begin{abstract}
In this paper, we prove some common fixed point results for four mappings satisfying generalized contractive condition in $S$-metric space. Our results extend and improve several previous works.
\end{abstract}

Keywords Common fixed point $\cdot S$-metric space $\cdot$ Compatible mappings

Mathematics Subject Classification $47 \mathrm{H} 10 \cdot 54 \mathrm{H} 25$

\section{Introduction and preliminaries}

Banach's contraction principle in metric spaces is one of the most important results in the theory of fixed points and non-linear analysis in general. From 1922, when Stefan Banach formulated the notion of contraction and proved the famous theorem, scientists around the world are publishing new results that are connected either to establish a generalization of metric space or to get a improvement of contractive condition. In the literature most famous improvement of contractive condition is certainly the papers of Edelstein-Nemytskii, Boyd-Wong, Meir-Keeler, Kannan, Chatterje, Zamfirescu, and certainly Ćirić.

In addition to the improvement of Banach's contractive conditions, more and more attention is devoted itself to the generalization of metric spaces such as 2-metric spaces,

T. Došenović

tatjanad@tf.uns.ac.rs

S. Sedghi

sedghi_gh@yahoo.com; sedghi.gh@qaemiau.ac.ir

N. Shobkolaei

nabi_shobe@yahoo.com

M. Shahraki

m.shahraki@gu.ac.ir

1 Department of Mathematics, Qaemshahr Branch, Islamic Azad University, Qaemshahr, Iran

2 Department of Mathematics, Babol Branch, Islamic Azad University, Babol, Iran

3 Faculty of Technology, University of Novi Sad, Bulevar Cara Lazara 1, Novi Sad, Serbia
$D *$-metric spaces, partial metric spaces, cone metric spaces, $b$-metric spaces, $G$-metric spaces and others. One of the generalization of metric spaces is given in the paper of Sedghi et al. [1]. They introduced a notion of $S$-metric spaces and give some of their properties. For more details regarding this spaces we refer [1-12].

For the sake of transparency, we list the basic properties of $S$-metric spaces that will be used later.

Definition 1.1 [1] Let $X$ be a nonempty set. A function $S: X^{3} \rightarrow[0, \infty)$ is said to be an $S$-metric on $X$, if for each $x, y, z, a \in X$,

1. $S(x, y, z)=0$ if and only if $x=y=z$,

2. $S(x, y, z) \leq S(x, x, a)+S(y, y, a)+S(z, z, a)$.

The pair $(X, S)$ is called an $S$-metric space.

Example 1.2 [1] We can easily check that the following examples are $S$-metric spaces.

1. Let $X=\mathbb{R}^{n}$ and $\|\cdot\|$ be a norm on $X$. Then $S(x, y, z)=$ $\|y+z-2 x\|+\|y-z\|$ is an $S$-metric on $X$. In general, if $X$ is a vector space over $\mathbb{R}$ and $\|\cdot\|$ is a norm on $X$. Then it is easy to see that

$S(x, y, z)=\|\alpha y+\beta z-\lambda x\|+\|y-z\|$,

where $\alpha+\beta=\lambda$ for every $\alpha, \beta \geq 1$, is an $S$-metric on $X$.

2. Let $X$ be a nonempty set and $d_{1}, d_{2}$ be two ordinary metrics on $X$. Then

$S(x, y, z)=d_{1}(x, z)+d_{2}(y, z)$,

is an $S$-metric on $X$. 
Lemma 1.3 [8] Let $(X, S)$ be an S-metric space. Then, we have $S(x, x, y)=S(y, y, x), x, y \in X$.

Definition 1.4 [9] Let $(X, S)$ be an $S$-metric space and $A \subset X$.

1. A sequence $\left\{x_{n}\right\}$ in $X$ converges to $x$ if $S\left(x_{n}, x_{n}, x\right) \rightarrow 0$ as $n \rightarrow \infty$, that is for every $\varepsilon>0$ there exists $n_{0} \in \mathbb{N}$ such that for $n \geq n_{0}, S\left(x_{n}, x_{n}, x\right)<\varepsilon$. This case, we denote by $\lim _{n \rightarrow \infty} x_{n}=x$ and we say that $x$ is the limit of $\left\{x_{n}\right\}$ in $X$.

2. A sequence $\left\{x_{n}\right\}$ in $X$ is said to be Cauchy sequence if for each $\varepsilon>0$, there exists $n_{0} \in \mathbb{N}$ such that $S\left(x_{n}, x_{n}, x_{m}\right)<\varepsilon$ for each $n, m \geq n_{0}$.

3. The $S$-metric space $(X, S)$ is said to be complete if every Cauchy sequence is convergent.

Definition 1.5 [6] Let $(X, S)$ and $\left(X^{\prime}, S^{\prime}\right)$ be two $S$-metric spaces, and let $f:(X, S) \rightarrow\left(X^{\prime}, S^{\prime}\right)$ be a function. Then $f$ is said to be continuous at a point $a \in X$ if and only if for every sequence $x_{n}$ in $X, S\left(x_{n}, x_{n}, a\right) \rightarrow 0$ implies $S^{\prime}\left(f\left(x_{n}\right), f\left(x_{n}\right), f(a)\right) \rightarrow 0$. A function $f$ is continuous at $X$ if and only if it is continuous at all $a \in X$.

Lemma 1.6 [9] Let $(X, S)$ be an S-metric space. If there exist sequences $\left\{x_{n}\right\}$ and $\left\{y_{n}\right\}$ such that $\lim _{n \rightarrow \infty} x_{n}=x$ and $\lim _{n \rightarrow \infty} y_{n}=y$, then

$\lim _{n \rightarrow \infty} S\left(x_{n}, x_{n}, y_{n}\right)=S(x, x, y)$.

Definition 1.7 Let $(X, S)$ be an $S$-metric space. A pair $\{f, g\}$ is said to be compatible if and only if $\lim _{n \rightarrow \infty} S\left(f g x_{n}, f g x_{n}, g f x_{n}\right)=0$, whenever $\left\{x_{n}\right\}$ is a sequence in $X$ such that $\lim _{n \rightarrow \infty} f x_{n}=\lim _{n \rightarrow \infty} g x_{n}=t$ for some $t \in X$.

\section{Common fixed point results}

We start with the following lemma.

Lemma 2.1 Let $(X, S)$ be an $S$-metric space. If there exists two sequences $\left\{x_{n}\right\}$ and $\left\{y_{n}\right\}$ such that $\lim _{n \rightarrow \infty}$ $S\left(x_{n}, x_{n}, y_{n}\right)=0$, whenever $\left\{x_{n}\right\}$ is a sequence in $X$ such that $\lim _{n \rightarrow \infty} x_{n}=t$ for some $t \in X$, then $\lim _{n \rightarrow \infty} y_{n}=t$.

Proof By the triangle inequality in $S$-metric space, we have

$S\left(y_{n}, y_{n}, t\right) \leq 2 S\left(y_{n}, y_{n}, x_{n}\right)+S\left(t, t, x_{n}\right)$.

Now, by taking the upper limit when $n \rightarrow \infty$ in above inequality we get

$\limsup _{n \longrightarrow \infty} S\left(y_{n}, y_{n}, t\right) \leq 2 \limsup _{n \longrightarrow \infty} S\left(y_{n}, y_{n}, x_{n}\right)+\limsup _{n \longrightarrow \infty} S\left(t, t, x_{n}\right)=0$.
Hence $\lim _{n \rightarrow \infty} y_{n}=t$.

Theorem 2.2 Suppose that $f, g, R$ and $T$ are self maps of a complete S-metric space $(X, S)$, with $f(X) \subseteq T(X), g(X) \subseteq$ $R(X)$ and that the pairs $\{f, R\}$ and $\{g, T\}$ are compatible. If

$$
\begin{aligned}
& S(f x, f y, g z) \leq q \max \{S(R x, R y, T z), S(f x, f x, R x), \\
& \quad S(g z, g z, T z), S(f y, f y, g z)\},
\end{aligned}
$$

for each $x, y, z \in X$, with $0<q<1$.

Then $f, g, R$ and $T$ have a unique common fixed point in $X$ provided that $R$ and $T$ are continuous.

Proof Let $x_{0} \in X$. Since $f(X) \subseteq T(X)$, there exists $x_{1} \in X$ such that $f x_{0}=T x_{1}$, and also as $g x_{1} \in R(X)$, we choose $x_{2} \in X$ such that $g x_{1}=R x_{2}$. In general, $x_{2 n+1} \in X$ is chosen such that $f x_{2 n}=T x_{2 n+1}$ and $x_{2 n+2} \in X$ such that $g x_{2 n+1}=R x_{2 n+2}$, we obtain a sequence $\left\{y_{n}\right\}$ in $X$ such that

$y_{2 n}=f x_{2 n}=T x_{2 n+1}$,

$y_{2 n+1}=g x_{2 n+1}=R x_{2 n+2}, \quad n \geq 0$.

Now, we show that $\left\{y_{n}\right\}$ is a Cauchy sequence. For this we have

$$
\begin{aligned}
S\left(y_{2 n}, y_{2 n}, y_{2 n+1}\right)= & S\left(f x_{2 n}, f x_{2 n}, g x_{2 n+1}\right) \\
\leq & q \max \left\{S\left(R x_{2 n}, R x_{2 n}, T x_{2 n+1}\right),\right. \\
& S\left(f x_{2 n}, f x_{2 n}, R x_{2 n}\right), \\
& S\left(g x_{2 n+1}, g x_{2 n+1}, T x_{2 n+1}\right), \\
& \left.S\left(f x_{2 n}, f x_{2 n}, g x_{2 n+1}\right)\right\} \\
= & q \max \left\{S\left(y_{2 n-1}, y_{2 n-1}, y_{2 n}\right), S\left(y_{2 n}, y_{2 n}, y_{2 n-1}\right),\right. \\
& S\left(y_{2 n+1}, y_{2 n+1}, y_{2 n}\right), \\
& \left.S\left(y_{2 n}, y_{2 n}, y_{2 n+1}\right)\right\} \\
= & q \max \left\{S\left(y_{2 n-1}, y_{2 n-1}, y_{2 n}\right), S\left(y_{2 n}, y_{2 n}, y_{2 n+1}\right)\right\} .
\end{aligned}
$$

Now, if $S\left(y_{2 n}, y_{2 n}, y_{2 n+1}\right)>S\left(y_{2 n-1}, y_{2 n-1}, y_{2 n}\right)$, then by above inequality we have

$S\left(y_{2 n}, y_{2 n}, y_{2 n+1}\right)<q S\left(y_{2 n}, y_{2 n}, y_{2 n+1}\right)$,

which is a contradiction. Hence, $S\left(y_{2 n}, y_{2 n}, y_{2 n+1}\right) \leq$ $S\left(y_{2 n-1}, y_{2 n-1}, y_{2 n}\right)$, therefore by above inequality we get

$S\left(y_{2 n}, y_{2 n}, y_{2 n+1}\right) \leq q S\left(y_{2 n-1}, y_{2 n-1}, y_{2 n}\right)$.

By similar arguments we have: 


$$
\begin{aligned}
S\left(y_{2 n-1}, y_{2 n-1}, y_{2 n}\right)= & S\left(y_{2 n}, y_{2 n}, y_{2 n-1}\right) \\
= & S\left(f x_{2 n}, f x_{2 n}, g x_{2 n-1}\right) \\
\leq & q \max \left\{S\left(R x_{2 n}, R x_{2 n}, T x_{2 n-1}\right),\right. \\
& S\left(f x_{2 n}, f x_{2 n}, R x_{2 n}\right), \\
& S\left(g x_{2 n-1}, g x_{2 n-1}, T x_{2 n-1}\right), \\
& \left.S\left(f x_{2 n}, f x_{2 n}, g x_{2 n-1}\right)\right\} \\
= & q \max \left\{S\left(y_{2 n-1}, y_{2 n-1}, y_{2 n-2}\right),\right. \\
& S\left(y_{2 n}, y_{2 n}, y_{2 n-1}\right), \\
& S\left(y_{2 n-1}, y_{2 n-1}, y_{2 n-2}\right), \\
& \left.S\left(y_{2 n}, y_{2 n}, y_{2 n-1}\right)\right\} \\
= & q \max \left\{S\left(y_{2 n-2}, y_{2 n-2}, y_{2 n-1}\right),\right. \\
& \left.S\left(y_{2 n}, y_{2 n}, y_{2 n-1}\right)\right\} .
\end{aligned}
$$

Now, if $S\left(y_{2 n}, y_{2 n}, y_{2 n-1}\right)>S\left(y_{2 n-2}, y_{2 n-2}, y_{2 n-1}\right)$, then by above inequality we have

$$
S\left(y_{2 n}, y_{2 n}, y_{2 n-1}\right)<q S\left(y_{2 n}, y_{2 n}, y_{2 n-1}\right),
$$

which is a contradiction. Hence, $S\left(y_{2 n-1}, y_{2 n-1}, y_{2 n}\right) \leq$ $S\left(y_{2 n-2}, y_{2 n-2}, y_{2 n-1}\right)$, therefore by above inequality we get

$$
S\left(y_{2 n-1}, y_{2 n-1}, y_{2 n}\right) \leq q S\left(y_{2 n-2}, y_{2 n-2}, y_{2 n-1}\right) .
$$

Now, from (2) and (3) we have

$$
S\left(y_{n}, y_{n}, y_{n-1}\right) \leq q S\left(y_{n-1}, y_{n-1}, y_{n-2}\right), \quad n \geq 2
$$

where $0<q<1$.

Hence, for $n \geq 2$ it follows that

$$
S\left(y_{n}, y_{n}, y_{n-1}\right) \leq \cdots \leq q^{n-1} S\left(y_{1}, y_{1}, y_{0}\right) \text {. }
$$

By the triangle inequality in $S$-metric space, for $n>m$ we have

$$
\begin{aligned}
S\left(y_{n}, y_{n}, y_{m}\right) \leq & 2 S\left(y_{m}, y_{m}, y_{m+1}\right)+2 S\left(y_{m+1}, y_{m+1}, y_{m+2}\right) \\
& +\cdots+S\left(y_{n-1}, y_{n-1}, y_{n}\right) \\
< & 2 S\left(y_{m}, y_{m}, y_{m+1}\right)+2 S\left(y_{m+1}, y_{m+1}, y_{m+2}\right) \\
& +\cdots+2 S\left(y_{n-1}, y_{n-1}, y_{n}\right) .
\end{aligned}
$$

Hence from (4) and as $0<q<1$ we have

$$
\begin{aligned}
S\left(y_{n}, y_{n}, y_{m}\right) & \leq 2\left(q^{m}+q^{m+1}+\cdots+q^{n-1}\right) S\left(y_{1}, y_{1}, y_{0}\right) \\
& \leq 2 q^{m}\left[1+q+q^{2}+\cdots\right] S\left(y_{1}, y_{1}, y_{0}\right) \\
& \leq 2 \frac{q^{m}}{1-q} S\left(y_{1}, y_{1}, y_{0}\right) \rightarrow 0, \quad \text { as } \quad m \rightarrow \infty .
\end{aligned}
$$

It follows that $\left\{y_{n}\right\}$ is a Cauchy sequence. Since $X$ is a complete $S$-metric space, there is some $y$ in $X$ such that

$\lim _{n \rightarrow \infty} f x_{2 n}=\lim _{n \rightarrow \infty} T x_{2 n+1}=\lim _{n \rightarrow \infty} g x_{2 n+1}=\lim _{n \rightarrow \infty} R x_{2 n+2}=y$.

We show that $y$ is a common fixed point of $f, g, R$ and $T$.

Since $R$ is continuous it follows that $\lim _{n \rightarrow \infty} R^{2} x_{2 n+2}=R y, \quad \lim _{n \rightarrow \infty} R f x_{2 n}=R y$.

And since $f$ and $R$ are compatible, $\lim _{n \rightarrow \infty} S\left(f R x_{2 n}, f R x_{2 n}\right.$, $\left.R f x_{2 n}\right)=0$. So by Lemma $2.1 \lim _{n \rightarrow \infty} f R x_{2 n}=R y$.

Putting $x=y=R x_{2 n}$ and $z=x_{2 n+1}$ in condition (1), we obtain

$$
\begin{aligned}
S\left(f R x_{2 n}, f R x_{2 n}, g x_{2 n+1}\right) \leq & q \max \left\{S\left(R^{2} x_{2 n}, R^{2} x_{2 n}, T x_{2 n+1}\right),\right. \\
& S\left(f R x_{2 n}, f R x_{2 n}, R^{2} x_{2 n}\right), \\
& S\left(g x_{2 n+1}, g x_{2 n+1}, T x_{2 n+1}\right), \\
& \left.S\left(f R x_{2 n}, f R x_{2 n}, g x_{2 n+1}\right)\right\} .
\end{aligned}
$$

Now, by taking the upper limit when $n \rightarrow \infty$ in (5) we get

$$
\begin{aligned}
S(R y, R y, y)= & \lim _{n \longrightarrow \infty} S\left(f R x_{2 n}, f R x_{2 n}, g x_{2 n+1}\right) \\
\leq & q \max _{n \longrightarrow \infty} S\left(\lim ^{2} x_{2 n}, R^{2} x_{2 n}, T x_{2 n+1}\right), \\
& \lim _{n \rightarrow \infty} S\left(f R x_{2 n}, f R x_{2 n}, R^{2} x_{2 n}\right), \\
& \lim _{n \rightarrow \infty} S\left(g x_{2 n+1}, g x_{2 n+1}, T x_{2 n+1}\right), \\
& \left.\lim _{n \longrightarrow \infty} S\left(f R x_{2 n}, f R x_{2 n}, g x_{2 n+1}\right)\right\} . \\
\leq & q \max \{S(R y, R y, y), 0,0, S(R y, R y, y))\} \\
= & q S(R y, R y, y) .
\end{aligned}
$$

Consequently, $S(R y, R y, y) \leq q S(R y, R y, y)$, as $0<q<1$ it follows that $R y=y$.

In a similar way, since $T$ is continuous, we obtain that $\lim _{n \rightarrow \infty} T^{2} x_{2 n+1}=T y, \quad \lim _{n \rightarrow \infty} T g x_{2 n+1}=T y$.

Since $g$ and $T$ are compatible, $\lim _{n \rightarrow \infty} S\left(g T x_{2 n+1}, g T x_{2 n+1}\right.$, $\left.T g x_{2 n+1}\right)=0$. So by Lemma $2.1 \lim _{n \rightarrow \infty} g T x_{2 n+1}=T y$.

Putting $x=y=x_{2 n}$ and $z=T x_{2 n+1}$ in condition (1), we obtain

$$
\begin{aligned}
S\left(f x_{2 n}, f x_{2 n}, g T x_{2 n+1}\right) \leq & q \max \left\{S\left(R x_{2 n}, R x_{2 n}, T^{2} x_{2 n+1}\right),\right. \\
& S\left(f x_{2 n}, f x_{2 n}, R x_{2 n}\right) \\
& S\left(g T x_{2 n+1}, g T x_{2 n+1}, T^{2} x_{2 n+1}\right) \\
& \left.S\left(f x_{2 n}, f x_{2 n}, g T x_{2 n+1}\right)\right\}
\end{aligned}
$$

Similarly by taking the upper limit when $n \rightarrow \infty$ in (6), we obtain

$$
\begin{aligned}
S(y, y, T y)= & \lim _{n \longrightarrow \infty} S\left(f x_{2 n}, f x_{2 n}, g T x_{2 n+1}\right) \\
& \leq q \max \{S(y, y, T y), 0,0, S(y, y, T y))\},
\end{aligned}
$$

that is, again it follows that $T y=y$. Also, we can apply condition (1) to obtain 
$S\left(f y, f y, g x_{2 n+1}\right) \leq q \max \left\{S\left(R y, R y, T x_{2 n+1}\right), S(f y, f y, R y)\right.$,

$$
\left.S\left(g x_{2 n+1}, g x_{2 n+1}, T x_{2 n+1}\right), S\left(f y, f y, g x_{2 n+1}\right)\right\} \text {. }
$$

And by taking the upper limit when $n \rightarrow \infty$ in (7), as $R y=T y=y$, we have

$$
\begin{aligned}
S(f y, f y, y) & \leq q \max \{S(R y, R y, y), S(f y, f y, y), S(y, y, y), S(f y, f y, y)\} \\
& =q S(f y, f y, y) .
\end{aligned}
$$

Since $0<q<1$, it follows that $S(f y, f y, y)=0$ and $f y=y$.

Finally by using of condition (1) and as, $R y=T y=$ $f y=y$, we obtain

$$
\begin{aligned}
S(y, y, g y)= & S(f y, f y, g y) \\
\leq & q \max \{S(R y, R y, T y), S(f y, f y, R y), \\
& S(g y, g y, T y), S(f y, f y, g y)\} \\
= & q S(y, y, g y),
\end{aligned}
$$

which implies that $S(y, y, g y)=0$ and $g y=y$.

Thus we proved that

$R y=T y=f y=g y=y$.

If there exists another common fixed point $x$ in $X$ of all $f, g, R$ and $T$, then

$$
\begin{aligned}
S(x, x, y)=S(f x, f x, g y) & \\
\leq & q \max \{S(R x, R x, T y), S(f x, f x, R x), \\
& S(g y, g y, T y), S(f x, f x, g y)\} \\
= & q \max \{S(x, x, y), S(x, x, x), \\
& S(y, y, y), S(x, x, y)\} \\
= & q S(x, x, y),
\end{aligned}
$$

which implies that $S(x, x, y)=0$ and $x=y$. Thus $y$ is a unique common fixed point of $f, g, R$ and $T$. The proof of the theorem is completed.

Now we give an example to support our result.

Example 2.3 Let $X=[0,1]$ be endowed with $S$-metric $S(x, y, z)=|x-z|+|y-z|$. Define $f, g, R$ and $T$ on $X$ by

$$
\begin{gathered}
f(x)=\left(\frac{x}{2}\right)^{8}, \quad g(x)=\left(\frac{x}{2}\right)^{4}, \\
R(x)=\left(\frac{x}{2}\right)^{2}, \quad T(x)=\frac{x}{2} .
\end{gathered}
$$

Obviously $f(X) \subseteq T(X)$ and $g(X) \subseteq R(X)$. Furthermore, the pairs $\{f, R\}$, and $\{g, T\}$ are compatible mappings.

Also for each $x, y, z \in X$, we have

$$
\begin{aligned}
S(f x, f y, g z)= & |f x-g z|+|f y-g z| \\
= & \left|\left(\frac{x}{2}\right)^{8}-\left(\frac{z}{2}\right)^{4}\right|+\left|\left(\frac{y}{2}\right)^{8}-\left(\frac{z}{2}\right)^{4}\right| \\
= & \left|\left(\frac{x}{2}\right)^{4}-\left(\frac{z}{2}\right)^{2}\right|\left|\left(\frac{x}{2}\right)^{4}+\left(\frac{z}{2}\right)^{2}\right| \\
& +\left|\left(\frac{y}{2}\right)^{4}-\left(\frac{z}{2}\right)^{2}\right|\left|\left(\frac{y}{2}\right)^{4}+\left(\frac{z}{2}\right)^{2}\right| \\
\leq & \frac{5}{16}\left|\left(\frac{x}{2}\right)^{2}-\frac{z}{2}\right|\left|\left(\frac{x}{2}\right)^{2}+\frac{z}{2}\right|+\frac{5}{16}\left|\left(\frac{y}{2}\right)^{2}-\frac{z}{2}\right|\left|\left(\frac{y}{2}\right)^{2}+\frac{z}{2}\right| \\
\leq & \frac{15}{64}|R x-T z|+\frac{15}{64}|R y-T z|=\frac{15}{64} S(R x, R y, T z) \\
\leq & \frac{15}{64} \max \{S(R x, R y, T z), S(f x, f x, R x), \\
& S(g z, g z, T z), S(f y, f y, g z)\},
\end{aligned}
$$

where $\frac{15}{64} \leq q<1$. Thus $f, g, R$ and $T$ satisfy the conditions given in Theorem 2.2 and 0 is the unique common fixed point of $f, g, R$ and $T$.

Now we get the special cases of Theorem 2.2 as follows.

Corollary 2.4 Let $(X, S)$ be a complete $S$-metric space and let $f, g: X \rightarrow X$ be two mappings such that

$S(f x, f y, g z) \leq q \max \{S(x, y, z), S(f x, f x, x), S(g z, g z, z)$,

$S(f y, f y, g z)\}$, for all $x, y, z \in X$,

with $0<q<1$.

Then there exists a unique point $y \in X$ such that $f y=g y=y$.

Proof If we take $R$ and $T$ as identity maps on $X$, then Theorem 2.2 follows that $f$ and $g$ have a unique common fixed point.

Corollary 2.5 Let $(X, S)$ be a complete $S$-metric space and let $R, T: X \rightarrow X$ be continuous mappings onto $X$, such that

$S(x, y, z) \leq q \max \{S(R x, R y, T z), S(x, x, R x), S(z, z, T z)$,

$S(y, y, g z)\}$, for all $x, y, z \in X$,

with $0<q<1$.

Then $R$ and $T$ have a unique common fixed point.

Proof If we take $f$ and $g$ as identity maps on $X$, then from Theorem 2.2 follows that $S$ and $T$ have a unique common fixed point.

Corollary 2.6 Let $(X, S)$ be a complete $S$-metric space and let $f: X \rightarrow X$ be a mapping such that

$S(f x, f y, f z) \leq q \max \{S(x, y, z), S(f x, f x, x), S(f z, f z, z)$,

$S(f y, f y, f z)\}$, for all $x, y, z \in X$,

with $0<q<1$.

Then $f$ has a unique fixed point in $X$. 
Proof If we take $S$ and $T$ as identity maps on $X$ and $f=g$, then from Theorem 2.2 follows that $f$ have a unique common fixed point.

Theorem 2.7 Let $\{f, R\}$ and $\{g, T\}$ be compatible self mappings on a complete $S$-metric space $(X, S)$ and for all $x, y, z \in X$, satisfying

$$
\begin{aligned}
& S(f x, f y, g z) \leq a_{1} S(R x, R y, T z)+a_{2} S(f x, f x, T z) \\
& \quad+a_{3} S(R x, R y, g z)+a_{4} S(f y, f y, T z)+a_{5} S(g z, g z, T z),
\end{aligned}
$$

where $a_{i} \geq 0(i=1,2,3,4,5)$ are real constants with $a_{1}+$ $3 a_{2}+3 a_{3}+3 a_{4}+a_{5}<1$. If $f(X) \subseteq T(X)$ and $g(X) \subseteq$ $R(X)$ and $R$ and $T$ are continuous, then all $f, g, R$ and $T$ have a unique common fixed point.

Proof Let $x_{0}$ in $X$. Since $f(X) \subseteq T(X)$, let $x_{1} \in X$ be such that $T x_{1}=f x_{0}$, and also, as $g x_{1} \in R(x)$, let $x_{2} \in X$ be such that $R x_{2}=g x_{1}$. In general, $x_{2 n+1} \in X$ is chosen such that $T x_{2 n+1}=f x_{2 n}$ and $x_{2 n+2} \in X$ such that $R x_{2 n+2}=g x_{2 n+1}$; $n=0,1,2, \ldots$ Denote

$$
\begin{aligned}
y_{2 n} & =T x_{2 n+1}=f x_{2 n}, \\
y_{2 n+1} & =R x_{2 n+2}=g x_{2 n+1}, \quad n \geq 0 .
\end{aligned}
$$

Now, we show that $\left\{y_{n}\right\}$ is a Cauchy sequence. For this we have

$$
\begin{aligned}
S\left(y_{2 n}, y_{2 n}, y_{2 n+1}\right)= & S\left(f x_{2 n}, f x_{2 n}, g x_{2 n+1}\right) \\
\leq & a_{1} S\left(R x_{2 n}, R x_{2 n}, T x_{2 n+1}\right) \\
& +a_{2} S\left(f x_{2 n}, f x_{2 n}, T x_{2 n+1}\right) \\
& +a_{3} S\left(R x_{2 n}, R x_{2 n}, g x_{2 n+1}\right. \\
& +a_{4} S\left(f x_{2 n}, f x_{2 n}, T x_{2 n+1}\right) \\
& \left.+a_{5} S\left(g x_{2 n+1}, g x_{2 n+1}, T x_{2 n+1}\right)\right) \\
= & a_{1} S\left(y_{2 n-1}, y_{2 n-1}, y_{2 n}\right)+a_{2} S\left(y_{2 n}, y_{2 n}, y_{2 n}\right) \\
& +a_{3} S\left(y_{2 n-1}, y_{2 n-1}, y_{2 n+1}\right) \\
& +a_{4} S\left(y_{2 n}, y_{2 n}, y_{2 n}\right)+a_{5} S\left(y_{2 n+1}, y_{2 n+1}, y_{2 n}\right) \\
\leq & a_{1} S\left(y_{2 n-1}, y_{2 n-1}, y_{2 n}\right)+a_{3}\left[2 S\left(y_{2 n-1}, y_{2 n-1}, y_{2 n}\right)\right. \\
& \left.+S\left(y_{2 n+1}, y_{2 n+1}, y_{2 n}\right)\right]+a_{5} S\left(y_{2 n}, y_{2 n}, y_{2 n+1}\right) .
\end{aligned}
$$

Hence,

$$
\begin{aligned}
S\left(y_{2 n}, y_{2 n}, y_{2 n+1}\right) \leq & a_{1} S\left(y_{2 n-1}, y_{2 n-1}, y_{2 n}\right) \\
& +2 a_{3} S\left(y_{2 n-1}, y_{2 n-1}, y_{2 n}\right) \\
+ & \left(a_{3}+a_{5}\right) S\left(y_{2 n}, y_{2 n}, y_{2 n+1}\right) .
\end{aligned}
$$

Now we prove that $S\left(y_{2 n}, y_{2 n}, y_{2 n+1}\right) \leq S\left(y_{2 n-1}, y_{2 n-1}, y_{2 n}\right)$, for each $n \in \mathbb{N}$.

If $S\left(y_{2 n-1}, y_{2 n-1}, y_{2 n}\right)<S\left(y_{2 n}, y_{2 n}, y_{2 n+1}\right)$ for some $n \in$ $\mathbb{N}$, then from (9) we have

$$
\begin{aligned}
S\left(y_{2 n}, y_{2 n}, y_{2 n+1}\right) & <a_{1} S\left(y_{2 n}, y_{2 n}, y_{2 n+1}\right)+2 a_{3} S\left(y_{2 n}, y_{2 n}, y_{2 n+1}\right) \\
& +\left(a_{3}+a_{5}\right) S\left(y_{2 n}, y_{2 n}, y_{2 n+1}\right) \\
& =\left(a_{1}+3 a_{3}+a_{5}\right) S\left(y_{2 n}, y_{2 n}, y_{2 n+1}\right) \\
& <S\left(y_{2 n}, y_{2 n}, y_{2 n+1}\right)
\end{aligned}
$$

which is a contradiction.

So we have $S\left(y_{2 n}, y_{2 n}, y_{2 n+1}\right) \leq S\left(y_{2 n-1}, y_{2 n-1}, y_{2 n}\right)$, for each $n \in \mathbb{N}$ and from (9) we get

$S\left(y_{2 n}, y_{2 n}, y_{2 n+1}\right) \leq\left(a_{1}+3 a_{3}+a_{5}\right) S\left(y_{2 n-1}, y_{2 n-1}, y_{2 n}\right)$.

Also we have

$$
\begin{aligned}
S\left(y_{2 n-1}, y_{2 n-1}, y_{2 n}\right)= & S\left(y_{2 n}, y_{2 n}, y_{2 n-1}\right) \\
= & S\left(f x_{2 n}, f x_{2 n}, g x_{2 n-1}\right) \\
\leq & a_{1} S\left(R x_{2 n}, R x_{2 n}, T x_{2 n-1}\right) \\
& +a_{2} S\left(f x_{2 n}, f x_{2 n}, T x_{2 n-1}\right) \\
& +a_{3} S\left(R x_{2 n}, R x_{2 n}, g x_{2 n-1}\right) \\
& +a_{4} S\left(f x_{2 n}, f x_{2 n}, T x_{2 n-1}\right) \\
& +a_{5} S\left(g x_{2 n-1}, g x_{2 n-1}, T x_{2 n-1}\right) \\
= & a_{1} S\left(y_{2 n-1}, y_{2 n-1}, y_{2 n-2}\right) \\
& +a_{2} S\left(y_{2 n}, y_{2 n}, y_{2 n-2}\right) \\
& +a_{3} S\left(y_{2 n-1}, y_{2 n-1}, y_{2 n-1}\right) \\
& +a_{4} S\left(y_{2 n}, y_{2 n}, y_{2 n-2}\right) \\
& +a_{5} S\left(y_{2 n-1}, y_{2 n-1}, y_{2 n-2}\right) \\
\leq & a_{1} S\left(y_{2 n-1}, y_{2 n-1}, y_{2 n-2}\right) \\
& +\left(2 a_{2}+2 a_{4}\right) S\left(y_{2 n-1}, y_{2 n-1}, y_{2 n}\right) \\
& +\left(a_{2}+a_{4}\right) S\left(y_{2 n-1}, y_{2 n-1}, y_{2 n-2}\right) \\
& +a_{5} S\left(y_{2 n-1}, y_{2 n-1}, y_{2 n-2}\right) .
\end{aligned}
$$

Hence,

$$
\begin{aligned}
S\left(y_{2 n}, y_{2 n}, y_{2 n-1}\right) \leq & a_{1} S\left(y_{2 n-1}, y_{2 n-1}, y_{2 n-2}\right) \\
& +\left(2 a_{2}+2 a_{4}\right) S\left(y_{2 n}, y_{2 n}, y_{2 n-1}\right) \\
+ & \left(a_{2}+a_{4}+a_{5}\right) S\left(y_{2 n-1}, y_{2 n-1}, y_{2 n-2}\right) .
\end{aligned}
$$

Similarly, if $S\left(y_{2 n-1}, y_{2 n-1}, y_{2 n-2}\right)<S\left(y_{2 n}, y_{2 n}, y_{2 n-1}\right)$ for some $n \in \mathbb{N}$ then from (11), we obtain

$$
\begin{aligned}
S\left(y_{2 n}, y_{2 n}, y_{2 n-1}\right) & \leq\left(a_{1}+3 a_{2}+3 a_{4}+a_{5}\right) S\left(y_{2 n}, y_{2 n}, y_{2 n-1}\right) \\
& <S\left(y_{2 n}, y_{2 n}, y_{2 n-1}\right)
\end{aligned}
$$

which is a contradiction.

So we have $S\left(y_{2 n}, y_{2 n}, y_{2 n-1}\right) \leq S\left(y_{2 n-1}, y_{2 n-1}, y_{2 n-2}\right)$, for each $n \in \mathbb{N}$ and from (11) we get 


$$
\begin{aligned}
& S\left(y_{2 n}, y_{2 n}, y_{2 n-1}\right) \leq\left(a_{1}+3 a_{2}+3 a_{4}+a_{5}\right) \\
& \quad S\left(y_{2 n-1}, y_{2 n-1}, y_{2 n-2}\right) .
\end{aligned}
$$

Now, from (10) and (12), we have

$$
S\left(y_{n}, y_{n}, y_{n-1}\right) \leq \lambda S\left(y_{n-1}, y_{n-1}, y_{n-2}\right), \quad n \geq 2,
$$

where $\lambda=\min \left\{a_{1}+3 a_{3}+a_{5}, a_{1}+3 a_{2}+3 a_{4}+a_{5}\right\}$. We know that $\lambda \in(0,1)$.

Hence, for $n \geq 2$ it follows that

$$
S\left(y_{n}, y_{n}, y_{n-1}\right) \leq \cdots \leq \lambda^{n-1} S\left(y_{1}, y_{1}, y_{0}\right) \text {. }
$$

By the triangle inequality in $S$-metric space, for $n>m$ we have

$$
\begin{aligned}
& S\left(y_{n}, y_{n}, y_{m}\right) \leq 2 S\left(y_{m}, y_{m}, y_{m+1}\right)+2 S\left(y_{m+1}, y_{m+1}, y_{m+2}\right) \\
& \quad+\cdots+2 S\left(y_{n-1}, y_{n-1}, y_{n}\right) .
\end{aligned}
$$

Hence from (13) and as $\lambda<1$, we have

$$
\begin{aligned}
S\left(y_{n}, y_{n}, y_{m}\right) \leq & 2\left(\lambda^{m}+\lambda^{m+1}+\cdots+\lambda^{n-1}\right) S\left(y_{1}, y_{1}, y_{0}\right) \\
\leq & 2 \lambda^{m}\left[1+\lambda+(\lambda)^{2}+\cdots\right] S\left(y_{1}, y_{1}, y_{0}\right) \\
\leq & \frac{2 \lambda^{m}}{1-\lambda} S\left(y_{1}, y_{1}, y_{0}\right) \\
& =\frac{2 \lambda^{m}}{1-\lambda} S\left(y_{1}, y_{1}, y_{0}\right) \rightarrow 0 \quad \text { as } \quad m \rightarrow \infty .
\end{aligned}
$$

It follows that $\left\{y_{n}\right\}$ is a Cauchy sequence. Let $y \in X$ be such that

$\lim _{n \rightarrow \infty} f x_{2 n}=\lim _{n \rightarrow \infty} T x_{2 n+1}=\lim _{n \rightarrow \infty} g x_{2 n+1}=\lim _{n \rightarrow \infty} R x_{2 n+2}=y$.

Since $R$ is continuous it follows that

$$
\lim _{n \rightarrow \infty} R^{2} x_{2 n+2}=R y, \quad \lim _{n \rightarrow \infty} R f x_{2 n}=R y .
$$

And since $f$ and $R$ are compatible, $\lim _{n \rightarrow \infty} S\left(f R x_{2 n}\right.$, $\left.f R x_{2 n}, R f x_{2 n}\right)=0$. So by Lemma $2.1 \lim _{n \rightarrow \infty} f R x_{2 n}=R y$.

From (8) it follows that

$$
\begin{aligned}
S\left(f R x_{2 n}, f R x_{2 n}, g x_{2 n+1}\right) \leq & a_{1} S\left(R^{2} x_{2 n}, R^{2} x_{2 n}, T x_{2 n+1}\right) \\
& +a_{2} S\left(f R x_{2 n}, f R x_{2 n}, T x_{2 n+1}\right) \\
& +a_{3} S\left(R^{2} x_{2 n}, R^{2} x_{2 n}, g x_{2 n+1}\right) \\
& +a_{4} S\left(f R x_{2 n}, f R x_{2 n}, T x_{2 n+1}\right) \\
& +a_{5} S\left(g x_{2 n+1}, g x_{2 n+1}, T x_{2 n+1}\right) .
\end{aligned}
$$

Taking the upper limit as $n \rightarrow \infty$, we get

$$
\begin{aligned}
S(R y, R y, y) \leq & a_{1} S(R y, R y, y)+a_{2} S(R y, R y, y)+a_{3} S(R y, R y, y) \\
& +a_{4} S(R y, R y, y)+a_{5} S(y, y, y) \\
\leq & a_{1} S(R y, R y, y)+a_{2} S(R y, R y, y)+a_{3} S(R y, R y, y) \\
& +a_{4} S(R y, R y, y) \\
= & \left(a_{1}+a_{2}+a_{3}+a_{4}\right) S(R y, R y, y) \\
\leq & \left(a_{1}+a_{2}+a_{3}+a_{4}+a_{5}\right) S(R y, R y, y) .
\end{aligned}
$$

Therefore $\quad S(R y, R y, y) \leq\left(a_{1}+3 a_{2}+3 a_{3}+3 a_{4}+a_{5}\right)$ $S(R y, R y, y)$, as $a_{1}+3 a_{2}+3 a_{3}+3 a_{4}+a_{5}<1$, we know that $R y=y$.

In a similar way, since $T$ is continuous, we obtain that $\lim _{n \rightarrow \infty} T^{2} x_{2 n+1}=T y, \quad \lim _{n \rightarrow \infty} \operatorname{Tg} x_{2 n+1}=T y$.

Since $g$ and $T$ are compatible, $\lim _{n \rightarrow \infty} S\left(g T x_{2 n+1}, g T x_{2 n+1}\right.$, $\left.T g x_{2 n+1}\right)=0$. So by Lemma $2.1 \lim _{n \rightarrow \infty} g T x_{2 n+1}=T y$.

From (8), it follows that

$$
\begin{aligned}
S\left(f x_{2 n}, f x_{2 n}, g T_{2 n+1}\right) & \leq a_{1} S\left(R x_{2 n}, R x_{2 n}, T^{2} x_{2 n+1}\right) \\
& +a_{2} S\left(f x_{2 n}, f x_{2 n}, T^{2} x_{2 n+1}\right) \\
& +a_{3} S\left(R x_{2 n}, R x_{2 n}, g T x_{2 n+1}\right) \\
& +a_{4} S\left(f x_{2 n}, f x_{2 n}, T^{2} x_{2 n+1}\right) \\
& +a_{5} S\left(g T x_{2 n+1}, g T x_{2 n+1}, T^{2} x_{2 n+1}\right) .
\end{aligned}
$$

Taking the upper limit as $n \rightarrow \infty$, we get

$$
\begin{aligned}
S(y, y, T y) \leq & a_{1} S(y, y, T y)+a_{2} S(y, y, T y)+a_{3} S(y, y, T y) \\
& +a_{4} S(y, y, T y)+a_{5} S(T y, T y, T y) \\
= & \left(a_{1}+a_{2}+a_{3}+a_{4}\right) S(y, y, T y) \\
\leq & \left(a_{1}+3 a_{2}+3 a_{3}+3 a_{4}+a_{5}\right) S(y, y, T y),
\end{aligned}
$$

that is,

$S(y, y, T y) \leq\left(a_{1}+3 a_{2}+3 a_{3}+3 a_{4}+a_{5}\right) S(y, y, T y)$.

Therefore, by $a_{1}+3 a_{2}+3 a_{3}+3 a_{4}+a_{5}<1$, we know that $T y=y$.

Again from (8), it follows that

$$
\begin{aligned}
S\left(f y, f y, g x_{2 n+1}\right) \leq & a_{1} S\left(R y, R y, T x_{2 n+1}\right)+a_{2} S\left(f y, f y, T x_{2 n+1}\right) \\
& +a_{3} S\left(R y, R y, g x_{2 n+1}\right)+a_{4} S\left(f y, f y, T x_{2 n+1}\right) \\
& \left.+a_{5} S\left(g x_{2 n+1}, g x_{2 n+1}, T x_{2 n+1}\right)\right) .
\end{aligned}
$$

And by taking the upper limit as $n \rightarrow \infty$, as $R y=y, T y=y$, we get

$$
\begin{aligned}
S(f y, f y, y) \leq & a_{1} S(y, y, y)+a_{2} S(f y, f y, y)+a_{3} S(y, y, y) \\
& +a_{4} S(f y, f y, y)+a_{5} S(y, y, y) \\
\leq & \left(a_{2}+a_{4}\right) S(f y, f y, y) .
\end{aligned}
$$

Therefore, $\quad S(f y, f y, y) \leq\left(a_{1}+3 a_{2}+3 a_{3}+3 a_{4}+\right.$ $\left.a_{5}\right) S(f y, f y, y)$ and by $a_{1}+3 a_{2}+3 a_{3}+3 a_{4}+a_{5}<1$, we know that $f y=y$. Again from (8) we have $S(f y, f y, g y)=0$; hence $f y=g y$. Thus we prove that $f y=g y=R y=T y=y$. If there exists another common fixed point $x$ in $X$ of all $f, g, R$ and $T$, then 


$$
\begin{aligned}
S(x, x, y) & =S(f x, f x, g y) \\
& \leq a_{1} S(R x, R x, T y)+a_{2} S(f x, f x, T y)+a_{3} S(R x, R x, g y) \\
& +a_{4} S(f x, f x, T y)+a_{5} S(g y, g y, T y) \\
& =\left(a_{1}+a_{2}+a_{3}+a_{4}\right) S(x, x, y) \\
& \leq\left(a_{1}+3 a_{2}+3 a_{3}+3 a_{4}+a_{5}\right) S(x, x, y) .
\end{aligned}
$$

From which it follows

$$
S(x, x, y) \leq\left(a_{1}+3 a_{2}+3 a_{3}+3 a_{4}+a_{5}\right) S(x, x, y) .
$$

Since $a_{1}+3 a_{2}+3 a_{3}+3 a_{4}+a_{5}<1$, it follows that $S(x, x, y)=0$, i.e., $x=y$. Therefore $y$ is a unique common fixed point of all $f, g, R$ and $T$. The proof of the theorem is completed.

Acknowledgements Funding was provided by MNTRSS (Grant No. 174009).

Open Access This article is distributed under the terms of the Creative Commons Attribution 4.0 International License (http://creative commons.org/licenses/by/4.0/), which permits unrestricted use, distribution, and reproduction in any medium, provided you give appropriate credit to the original author(s) and the source, provide a link to the Creative Commons license, and indicate if changes were made.

\section{References}

1. Sedghi, S., Shobe, N., Aliouche, A.: A generalization of fixed point theorems in $S$-metric spaces. Mat. Vesn. 64, 258-266 (2012)
2. Mojaradi, J.: Afra, double contraction in $S$-metric spaces. Int. J. Math. Anal. 9(3), 117-125 (2015)

3. Van Dunga, N., Trung Hieua, N., Radojević, S.: Fixed point theorems for $g$-monotone maps on partially ordered $S$-metric spaces. Filomat 28(9), 1885-1898 (2014)

4. Gholidahneh, A., Sedghi, S., Došenović, T., Radenović, S.: Ordered $S$-metric spaces and coupled common fixed point theorems of integral type contraction. Math. Interdiscip. Res. 2, 71-84 (2017)

5. Gupta, A.: Cyclic contraction on $S$-metric spaces. Int. J. Anal. Appl. 3(2), 119-130 (2013)

6. Kyu Kim, J., Sedghi, S., Gholidahneh, A., Mahdi Rezaee, M.: Fixed point theorems in $S$-metric spaces. East Asian Math. J. 32(5), 677-684 (2016)

7. Prudhvi, K.: Fixed point theorems in $S$-metric spaces. Univers. J. Comput. Math. 3(2), 19-21 (2015)

8. Sedghi, S., Altun, I., Shobe, N., Salahshour, M.A.: Some properties of $S$-metric spaces and fixed point results. Kyungpook Math. J. 54(1), 113-122 (2014)

9. Sedghi, S., Dung, N.V.: Fixed point theorems on $S$-metric spaces. Mat. Vesn. 66, 113-124 (2014)

10. Sedghi, S., Shobe, N., Došenović, T.: Fixed point results in $S$ metric spaces. Nonlinear Funct. Anal. Appl. 20(1), 55-67 (2015)

11. Sedghi, S., Došenović, T., Mahdi Rezaee, M., Radenović, S.: Common fixed point theorems for contractive mappings satisfying $\Phi$-maps in $S$-metric spaces. Acta Univ. Sapientiae Math. 8(2), 298311 (2016)

12. Sedghi, S., Gholidahneh, A., Došenović, T., Esfahani, J., Radenović, S.: Common fixed point of four maps in $S_{b}$-metric spaces. J. Linear Topol. Algebra 05(02), 93-104 (2016)

\section{Publisher's Note}

Springer Nature remains neutral with regard to jurisdictional claims in published maps and institutional affiliations. 